\title{
ESTUDIO DE RESIDUOS DE BOLDENONA EN CARNE DE BOVINOS FAENADOS EN EL CAMAL METROPOLITANO DE QUITO
}

\author{
Morales-Pérez, Elvis ${ }^{\mathrm{a}}$; Andrade-Beltrán, Paulette ${ }^{\mathrm{b}}$; Flores-Gutiérrez, Paola ${ }^{\mathrm{b}}$; Puga-Torres, Byron ${ }^{\mathrm{a} *}$; \\ De La Cueva-Jácome Francisco ${ }^{a}$, Vargas-Estrella, Javiera .

\begin{abstract}
${ }^{a}$ Universidad Central del Ecuador. Facultad de Medicina Veterinaria y Zootecnia. Jerónimo Leyton S/N y Gatto Sobral. Quito - Ecuador. Tumbaco. Quito-Ecuador.
\end{abstract} \\ ${ }^{\mathrm{b}}$ Agencia de Regulación y Control Fito y Zoosanitario Av. Interoceánica Km. 14 1⁄2, La Granja,
}

Ingresado: 10/12/2019

\section{Resumen}

Las necesidades de proteína de origen animal es un factor muy importante en el desarrollo biológico de los seres humanos, por lo cual deben cumplir requisitos importantes para que sean considerados inocuos y aptos para su consumo, puesto que en la crianza y producción de los animales, se utilizan varias sustancias farmacológicas como promotores de crecimiento y muchas veces no se cumplen los tiempos de retiro establecidos para que se eliminen estos fármacos de los tejidos comestibles, causando serias alteraciones en el consumidor, siendo más vulnerables los lactantes, niños pequeños, mujeres embarazadas y ancianos. Los objetivos del estudio estuvieron direccionados a realizar un levantamiento preliminar de información para determinar si existe presencia de boldenona en carne de bovinos faenados en el Camal Metropolitano de Quito y relacionar la presencia de ésta, en función a su edad y procedencia. Se tomaron 72 muestras de carne al azar, de animales con buena conformación, por un lapso de 4 semanas; posteriormente se analizaron por la técnica de screening de ELISA, en el Laboratorio de Contaminantes de Productos Pecuarios de AGROCALIDAD. Se determinó la presencia de residuos de boldenona en el $100 \%$ de las muestras analizadas, lo cual alerta sobre su posible uso como promotor de crecimiento en bovinos procedentes del litoral ecuatoriano, por lo que se debería analizar mediante técnicas confirmatorias como HPLC-MS para, de ser el caso,

\footnotetext{
* Correspondencia a: Facultad de Medicina Veterinaria y Zootecnia de la Universidad Central del Ecuador, Jerónimo Leyton s/n y Gatto Sobral, Quito - Ecuador. Teléfono: +593 2 2566160; Celular: +593 991906426. Correo electrónico: bpuga@uce.edu.ec
}

Aceptado: 31/03/2020

tomar las acciones preventivas y correctivas por parte de la Autoridad Nacional Competente.

Palabras clave: Boldenona, carne, ELISA, Quito

STUDY OF RESIDUES OF BOLDENONA IN BEEF FROM CATTLE SLAUGHTERED IN THE QUITO'S SLAUGHTERHOUSE
Abstract
The needs of animal protein is a very important factor in the human biological development, therefore, they must meet important requirements to be considered safe and suitable for consumption, since in the animal production, various pharmacological substances are used as growth promoters and many times the established withdrawal times are not fulfilled so that these drugs are eliminated from edible tissues, causing serious alterations in the consumer, being more vulnerable infants, young children, pregnant women and elderly. The objectives of the study were directed to carry out a preliminary information gathering to determine if there is presence of boldenone in meat of cattle slaughtered in the Slaughterhouse Metropolitan of Quito, and relate the presence of it, depending on their age and origin. 72 meat samples were taken at random, from animals with good conformation, for 4 weeks; later they were analyzed by the ELISA screening technique, at the AGROCALIDAD Livestock Products Contaminants Laboratory. The presence of boldenone residues was determined in $100 \%$ of the samples analyzed, which alerts its possible use as a growth promoter in cattle from the Ecuadorian 
coast, reason why it should be confirmed by confirmatory techniques such as HPLC-MS, and if necessary, take preventive and corrective actions by the Competent National Authority.

Keywords: Boldenone, meat, ELISA, Quito

\section{INTRODUCCIÓN}

La demanda de proteína de origen animal es uno de los elementos más preciados enfocados a la necesidad de mantener un adecuado desarrollo biológico de los seres humanos, [1] es por esto que la producción de carne para el consumo humano es uno de los factores más importantes dentro de la economía de un país, lo cual ha implicado el uso de promotores del crecimiento y dentro de éstos las hormonas esteroides, anabolizantes y otros, como fuentes para acelerar e incrementar la producción. [2] Al ser estos fármacos anabólicos una alternativa que permiten aumentar la producción cárnica [3] e inclusive llegan a ser usados ilegalmente por personas con la finalidad de superar sus condiciones atléticas, [4] especialmente la población más juvenil que los usa para mejorar su apariencia muscular y física, [5] corren el riesgo de sufrir un paro cardiaco [6], con hipertrofia ventricular izquierda. [7,8] Dentro de los anabólicos más usados en ganadería se encuentra el grupo de los esteroides, estrógenos y progestágenos, [9] siendo la boldenona un agente anabólico de acción prolongada [10] análogo de la testosterona, usado tanto en bovinos, equinos, porcinos, caprinos, caninos, [11] en aves por vía intramuscular, a quienes puede producir efectos hepatotóxicos y nefrotóxicos [12] y en conejos quienes tienen un aumento considerable en su peso y disminución de su conversión alimenticia, [13] existiendo la probabilidad de causarles alteraciones histopatológicas. [14]

La boldenona, como esteroide anabólico, ayuda en la generación de tejidos, al revertir el catabolismo de los mismos, inhibe a la testosterona por su propiedad de inhabilitar a la hormona luteinizante y estimula la eritropoyesis, [10] mejorando la ganancia diaria de peso, la eficiencia alimenticia y por ende el peso corporal final. [15] En la normativa ecuatoriana y en el Codex Alimentarius no se indican los Límites Máximos Permitidos de boldenona en carne de bovinos; existe autorización de uso solamente en equinos no destinados a consumo humano y en gatos con el fin de estimular el apetito, [10] sin embargo es prohibido su uso en varios deportes equinos [16] en los que se realiza constantes controles antidopaje. [17] Así mismo, su presencia en animales para consumo humano no es permitida, siendo prohibido en Argentina, Uruguay, Venezuela, Brasil, Paraguay, entre otros, mientras que en Colombia es autorizado con un tiempo de retiro de 30 días para carne. [2]

Diversos estudios realizados en torno a la presencia de boldenona en animales de abasto exponen resultados de su presencia en varios niveles y utilizando diferentes metodologías analíticas. [18-21] En algunos países ya no se consideran peligros potenciales, pues se encuentran en muy pequeñas concentraciones. [22] Sin embargo, en Ecuador existen escasos estudios publicados que determinen la presencia de residuos de boldenona en carne bovina, por lo cual el objetivo de esta investigación fue el evaluar su posible presencia, para que la autoridad competente desarrolle programas de farmacovigilancia que permitan garantizar la seguridad alimentaria de este producto de origen animal.

\section{METODOLOGÍA}

\section{Población y Muestra}

Como población objeto de estudio se seleccionaron bovinos procedentes del litoral ecuatoriano, los cuales fueron faenados en el Camal Metropolitano de Quito, ubicado en la Parroquia Ecuatoriana, Cantón Quito, Provincia Pichincha. Los bovinos seleccionados debían tener aptitud cárnica, estar entre 1 a 3 años de edad y con condición corporal de 6 a 9. Al final de la investigación se recolectaron muestras de 72 bovinos, los cuales fueron seleccionados por un muestreo aleatorio simple como método de randomización. [23]

La toma de muestras se realizó los días lunes, miércoles y viernes, entre mayo y junio del 2016, en el Camal Metropolitano de Quito. En la sala de sacrificio, se determinó la edad de los bovinos por medio de observación directa de la dentición, siguiendo las directrices respectivas. [24] Durante la inspección veterinaria de rutina, se procedió a tomar una muestra de 250 gramos del músculo semitendinoso, [25] el mismo que fue cubierto con papel aluminio para su preservación; posteriormente se los mantuvo en cadena de frío, mediante el uso de cooler con geles refrigerantes, para luego ser transportados al laboratorio, manteniendo una temperatura de $2-4^{\circ} \mathrm{C}$. Ya en el laboratorio se mantuvieron en congelación previo a su procesamiento y análisis.

\section{Método de Análisis}

El análisis y procesamiento de las muestras se 
realizó en el Laboratorio de Contaminantes de Productos Pecuarios de la Agencia Ecuatoriana de Aseguramiento de la Calidad del Agro AGROCALIDAD, ubicado en Tumbaco, Sector La Granja, Cantón Quito, Provincia de Pichincha.

La primera fase del análisis de laboratorio correspondió a la preparación de las muestras y la extracción del analito de interés, se redujo el tamaño de la muestra y se tomó una porción representativa para la determinación de Boldenona. La segunda fase del estudio consistió en la lectura de la placa. El método de análisis se lo realizó utilizando y siguiendo las instrucciones recomendadas en el Kit "MaxSignal ${ }^{\circledR}$ Boldenone ELISA Test" [Bioo Scientific Corporation, 7050 Burleson Rd - Austin, TX, USA], el cual se basa en el ensayo colorimétrico ELISA competitivo, para el análisis cuantitativo de boldenona, donde el analito de interés ha sido impregnado en los pocillos de la placa, luego la muestra es añadida en conjunto con el anticuerpo primario específico para el fármaco específico. El test tiene altas tasas de recuperación (>80\%), alta sensibilidad $(0,05 \mathrm{ng} / \mathrm{g}$ o ppb), bajo límite de detección $(0,025 \mathrm{ng} / \mathrm{g}$ o ppb) y alta reproducibilidad. [26]

\section{Preparación de reactivos}

Los reactivos se llevaron a temperatura ambiente antes de su uso. Para la preparación del 1X PBS, se mezcló 1 volumen del 10X PBS (que se encuentra en el kit) con 9 volúmenes de agua destilada. Para la preparación del tampón de equilibrio de la muestra $1 \mathrm{X}$, se añadió $10 \mathrm{~mL}$ de la sustancia tampón de equilibrio de la muestra I y $62 \mathrm{~g}$ del tampón de equilibrio de la muestra II (polvo), en una botella plástica de $250 \mathrm{~mL}$, luego se agregó 190 $\mathrm{ml}$ de agua destilada a la botella y se homogenizó correctamente.

El anticuerpo conjugado 1X HRP \#2 fue preparado mezclando 1 volumen del anticuerpo conjugado \#2 con 99 volúmenes del diluyente. La Solución de lavado $1 \mathrm{X}$ se consiguió al mezclar 1 volumen de solución de lavado $20 X$ con 19 volúmenes de agua destilada. Los estándares de boldenona $(0$ o control negativo, 0,05, 0,15, 0,5, 1,5, 4,5 y $10 \mathrm{ng} /$ $\mathrm{ml}$ ) se prepararon seriadamente a partir de la solución estándar con la concentración más alta hasta el estándar de la concentración más baja.

\section{Extracción del analito}

Las muestras se redujeron a fragmentos de 1 $\mathrm{cm}^{2}$, fueron molidas y se obtuvo un pool de cada muestra. De cada muestra homogenizada, se tomó $2 \mathrm{~g}$, a la cual se añadió $6 \mathrm{~mL}$ de Acetonitrilo y 2,5 mL de tampón de equilibrio de la muestra $1 X$. Luego de vorterizar por 3 minutos a velocidad máxima, se centrifugó la preparación por 10 minutos a 4000 x g (unidad de gravedad) y a temperatura ambiente $\left(20-25^{\circ} \mathrm{C}\right)$. Luego de transferir $3 \mathrm{~mL}$ del sobrenadante a un nuevo tubo, se añadió 300 mg de tejido limpio en la mezcla y nuevamente se vorterizó por 30 segundos. Le mezcla estuvo a temperatura ambiente por otros 5 minutos, para luego ser centrifugada por 10 minutos a $4000 \mathrm{x} \mathrm{g}$ a temperatura ambiente. $2,4 \mathrm{~mL}$ de sobrenadante fueron transferidos a un nuevo tubo, donde se secó la muestra con gas nitrógeno con rotavapor en un baño María de $50-60^{\circ} \mathrm{C}$, se añadió $300 \mu \mathrm{L}$ de $1 \mathrm{X}$ PBS, para luego vorterizar por 30 segundos a máxima velocidad. Finalmente se usó $50 \mu \mathrm{L}$ de la muestra para el ensayo.

\section{Prueba ELISA para Boldenona}

Se añadió $50 \mu \mathrm{L}$ de los estándares de boldenona, por duplicado, dentro de los primeros pocillos. Posteriormente $50 \mu \mathrm{L}$ de cada muestra, por duplicado, dentro de los restantes pocillos. En cada pocillo se añadió $100 \mu \mathrm{L}$ del anticuerpo \#1, homogenizando mediante un suave balanceado manual de la placa, durante 1 minuto. Luego de incubar la placa por 30 minutos a temperatura ambiente, se lavó la misma, por 3 ocasiones, con $250 \mu \mathrm{L}$ de solución de lavado; en el último lavado se invirtió la placa y se secó suavemente con una toalla de papel. Posteriormente se agregó $150 \mu \mathrm{L}$ de solución de anticuerpo \#2 (1X), para dejar incubar la placa por 30 minutos a temperatura ambiente y volver a lavar la placa, 3 veces, con $250 \mu \mathrm{L} 1 \mathrm{X}$ de solución de lavado. Nuevamente, luego del último lavado, se invirtió la placa y se secó suavemente con una toalla de papel. Se puso $100 \mu \mathrm{L}$ de sustrato $\mathrm{TMB}$, homogenizando manualmente la placa por 1 minuto. Finalmente, la placa fue incubada, por 15 minutos, a temperatura ambiente, luego de lo cual se añadió $100 \mu \mathrm{L}$ de la sustancia tampón de parada, con la finalidad de detener la reacción enzimática. La lectura de la placa se realizó inmediatamente en un lector de placas ELISA a una longitud de onda de 450 nanómetros (nm).

\section{Cálculo de la concentración de Boldenona}

Se usó el promedio de absorbancia relativo de cada muestra para determinar la concentración correspondiente de boldenona en $\mu \mathrm{g} / \mathrm{kg}$ y con esto se construyó una curva logarítmica, (Fig. 1) en base a los resultados de positividad y negatividad obtenidos del análisis, utilizando la fórmula: Absorbancia relativa $=($ absorbancia estándar $\mathrm{x}$ 100 / absorbancia del blanco).[26] 


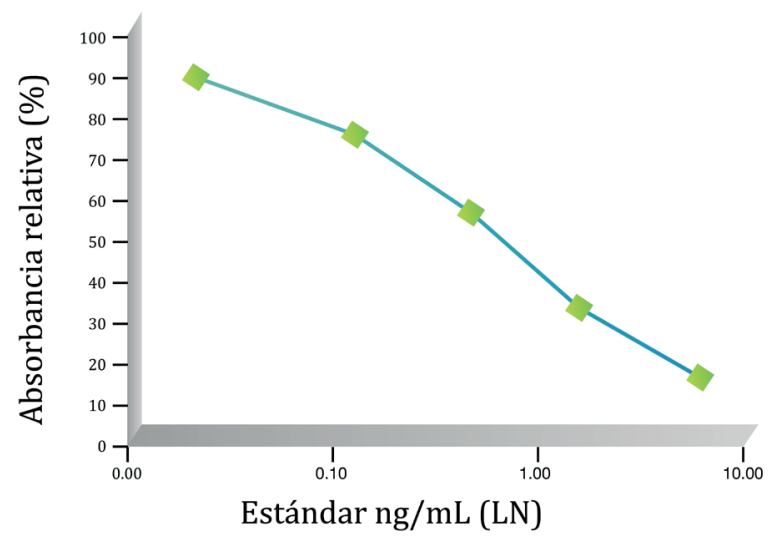

Fig. 1. Curva estándar de calibración del test ELISA competitivo para boldenona

Las pruebas ELISA son ampliamente usadas como pruebas de monitoreo, debido a su especificidad, rapidez y gran número de muestras que se analizan de forma simultánea, [27] con límites de detección entre 0,28 y $0,002 \mathrm{ng} / \mathrm{mL}$, [21] siendo el del test usado de 0,025 a $0,05 \mathrm{ng} / \mathrm{g}$ o ppb. [26]

\section{Análisis de datos}

Los datos obtenidos se registraron en Microsoft Excel. En el mismo programa se realizaron las tablas, gráficos y se expresaron los datos en porcentaje.

\section{RESULTADOS Y DISCUSIÓN}

Los resultados de la presente investigación sugieren la presencia de residuos de boldenona en el tejido muscular (carne), ya que resultaron positivas, por la prueba de screening de ELISA competitivo, el $100 \%$ de las muestras (72/72); el nivel más bajo encontrado fue de $0,2 \mu \mathrm{g} / \mathrm{kg}$ y el nivel más alto de $35 \mu \mathrm{g} / \mathrm{kg}$, lo cual podría deberse al uso de boldenona en bovinos procedentes del litoral con el fin de acelerar el crecimiento y producción de los animales.

De acuerdo a la procedencia de los animales (provincias 1, 2 y 3), de los 72 bovinos muestreados, el 68\% (49/72) proceden de la provincia 1, el 21\% (15/72) de la provincia 2, y el $11 \%(08 / 72)$ corresponde a la provincia 3 (Fig. 2). La concentración más baja fue de $0,2 \mu \mathrm{g} /$ $\mathrm{kg}$, encontrado en bovinos de la provincia $2 \mathrm{y} 1$, mientras que el valor más alto fue de $35,3 \mu \mathrm{g} / \mathrm{kg}$ procedente de la provincia 3 , lo cual al ser una concentración demasiada elevada se considera que pudo haber existido un secuestro farmacológico, es decir la acumulación exagerada del fármaco en un sitio del organismo, presumiblemente por administración del boldenona de 1 a 2 días antes de su sacrificio, realizado probablemente por negligencia o desconocimiento del operario. [28]

Animales muestreados en $\%$ por provincia

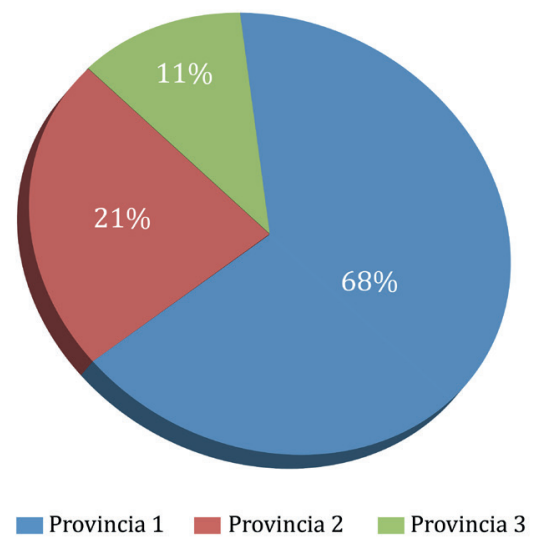

Fig. 2. Número de animales muestreados y su procedencia en el análisis de boldenona en bovinos de carne faenados en el Camal Metropolitano de Quito

De las 72 muestras positivas, el 87,5\% (63/72) corresponde a animales juveniles de 1 a 2 años; el otro 12,5\% (9/72) corresponde a bovinos adultos de 2 a 3 años de edad (Tabla 1). De los 49 animales procedentes de la provincia 1,44 fueron juveniles y 5 adultos; de la provincia 2,15 bovinos de los cuales 14 fueron juveniles y 1 adulto; finalmente de la provincia 3 provinieron 8 animales siendo 5 juveniles y 3 adultos. Esto nos indica que el uso de boldenona se realiza en los dos rangos de edad, debido a que el uso de sustancias de tipo hormonal, aceleran el crecimiento de los bovinos, gracias a su efecto anabólico; [7] así mismo, debido a la cantidad de residuos hallados en tejidos animales, se puede suponer que los animales estuvieron expuestos por periodos prolongados al principio activo [29] $\mathrm{y}$ que los agentes farmacológicos veterinarios se usan de forma reiterativa sin control técnico adecuado. [30]

Tabla 1: Distribución de los niveles de boldenona en bovinos de carne faenados en el Camal Metropolitano de Quito en función de la procedencia y su edad.

\begin{tabular}{|l|c|c|c|c|c|}
\cline { 2 - 6 } \multicolumn{1}{c|}{} & $\begin{array}{c}\text { Provincia } \\
\mathbf{1}\end{array}$ & $\begin{array}{c}\text { Provincia } \\
\mathbf{2}\end{array}$ & $\begin{array}{c}\text { Provincia } \\
\mathbf{3}\end{array}$ & $\begin{array}{c}\text { Número de } \\
\text { animales }\end{array}$ & Positivos \\
\hline $\begin{array}{l}\text { Juveniles } \\
\mathbf{( 1 - 2} \\
\text { años) }\end{array}$ & 44 & 14 & 5 & 63 & $100 \%$ \\
\hline $\begin{array}{l}\text { Adultos } \\
\mathbf{( 2 - 3} \\
\text { años) }\end{array}$ & 5 & 1 & 3 & 9 & $100 \%$ \\
\hline
\end{tabular}


Los valores del presente trabajo son extremadamente altos respecto al estudio realizado en carne de bovinos en Manabí, utilizando también la técnica de ELISA competitivo, donde se encontró que solamente el $14,6 \%$ de las muestras fueron positivas a boldenona. [31] Así mismo, en un estudio realizado en Colombia, usando la misma técnica de ELISA (tampoco se compararon mediante pruebas confirmatorias), el 23\% (25/111) de las muestras fueron consideradas no conformes, siendo positivas para boldenona con valores superiores al límite de detección de la prueba, atribuyéndose al no cumplimiento del tiempo de retiro, así como al uso de dosis superiores a las recomendadas, [32] siendo también valores bastante menores que los encontrados en esta investigación. Debido a esto se hace necesario la confirmación de los datos por pruebas confirmatorias como la Cromatografía Líquida de Alta Resolución acoplada a la espectrometría de masas (HPLC-MS).

Este trabajo debe representar una señal de alerta para la Autoridad Competente, pues la boldenona es un esteroide andrógeno anabólico usado para aumentar el crecimiento en animales de abasto de carne en muchos países del mundo, [33] sin embargo sus residuos pueden representar un potencial riesgo para los consumidores, [34] ya que puede causar efectos androgénicos como agresividad e inclusive hepatotoxicidad, [10] además que muchos promotores de crecimiento han sido catalogados por la Unión Europea como tóxicos y con propiedades cancerígenas. [35] Así mismo, puede ser un contaminante ambiental pues se puede encontrar en el estiércol de los animales junto con otros esteroides. [36]

\section{CONCLUSIONES}

En base a los resultados obtenidos en el presente estudio, mediante la prueba de screening de ELISA, se alerta sobre la posible presencia de residuos de boldenona en la carne de bovinos procedentes del litoral ecuatoriano y faenados en el Camal Metropolitano de Quito, lo que podría indicar su uso como promotor de crecimiento tanto en animales juveniles como en adultos, por lo que se debe confirmar esta presencia mediante el uso de una técnica confirmatoria como HPLCMS, para que la autoridad competente desarrolle programas de farmacovigilancia que garanticen la seguridad alimentaria de este producto de origen animal y de primera necesidad, realizando el análisis de un número importante de carne en mataderos de Ecuador, destinando los recursos económicos y humanos para dicho fin, capacitando al personal de laboratorio sobre esta problemática, y concientizando a los ganaderos sobre las consecuencias del uso de este tipo de productos en los consumidores, mediante campañas informativas y de sensibilización.

\section{AGRADECIMIENTOS}

A la Facultad de Medicina Veterinaria y Zootecnia de la Universidad Central del Ecuador, al DepartamentoVeterinario del Camal Metropolitano de Quito y al Laboratorio de Contaminantes de Productos Pecuarios de AGROCALIDAD.

\section{REFERENCIAS}

[1] FAO. El papel de la FAO en la sanidad animal [Internet]. Sanidad animal. 2019. Disponible en: http://www.fao.org/animal-health/es/

[2] Fajardo-Zapata ÁL, Méndez-Casallas FJ, Molina LH. Residuos de fármacos anabolizantes en carnes destinadas al consumo humano. Univ Sci [Internet]. 2011;16(1):77-91. Disponible en: http://www.scielo.org.co/pdf/unsc/v16n1/ v16n1a07.pdf

[3] Rairner S. Hormones in meat: different approachesintheEUand intheUSA.APMIS [Internet]. julio de 2001;109(S103):S357-64. Disponible en: http://doi.wiley.com/10.1111/j.1600-0463.2001. tb05787.x

[4] Laudo C, Puigdevall V, Del Río MJ, Velasco A, Pardos CL. Hormonas utilizadas como agentes ergogénicos: situación actual del problema Hormones used as ergogenics: present state of the question Aceptado para su publicación el 4 de noviembre de 2005. Correspondencia. An Sist Sanit Navar [Internet]. 2006;29(2):207-18. Disponible en: http://scielo.isciii.es/pdf/asisna/v29n2/ revision1.pdf[5] Singh V, Batta A. Suspected reactivation of extrapulmonary tuberculosis focus after non-medical abuse of anabolic androgenic steroids: a case report. J Basic Clin Physiol Pharmacol [Internet]. 28 de enero de 2019;31(1):12-25. Disponible en: http://www. degruyter.com/view/j/jbcpp.2020.31.issue-1/ jbcpp-2019-0167/jbcpp-2019-0167.xml

[6] Díaz M, Brito D, Gibrant F, Hernández R. Paro cardiaco repentino atribuido a miocardiopatía hipertrófica por uso de esteroides anabólicos. Reporte de caso. Med Crítica [Internet]. 2017;31(2):101-5. Disponible en: http://www. scielo.org.mx/pdf/mccmmc/v31n2/2448-8909mccmmc-31-02-101.pdf

[7] Park M, Sim J, Jeon Y, Yeon S, Lee J, In S. Determination of boldenone in postmortem specimens including blood and urine samples 
using LC-MS/MS. J Pharm Biomed Anal [Internet]. mayo de 2019;169:111-5. Disponible en: https://linkinghub.elsevier.com/retrieve/pii/ S073170851831882X

[8] Lehmann S, Thomas A, Schiwy-Bochat $\mathrm{K}-\mathrm{H}$, Geyer H, Thevis M, Glenewinkel F, et al. Death after misuse of anabolic substances (clenbuterol, stanozolol and metandienone). Forensic Sci Int [Internet]. octubre de 2019;303:109925. Disponible en: https://linkinghub.elsevier.com/ retrieve/pii/S0379073819303378

[9] Gasque R. Enciclopedia Bovina [Internet]. México UA de., editor. México DF: Mc Graw-Hill; 2008. Disponible en: https://www.academia. edu/8275187/Enciclopedia_Bovina_UNAM

[10] Plumb D. Veterinaria, Manual de Farmacología [Internet]. Sexta Edic. Inter-Médica, editor. Buenos Aires - Argentina; 2010. 134-134 p. Disponible en: http://libros-medicina-veterinaria. blogspot.com/2016/10/manual-de-farmacologiaveterinaria-pdf.html

[11] Larrea F, Chirinos M. Impacto en el humano de aditivos hormonales empleados en bovinos productores de carne. Rev Investig Clínica [Internet]. 2007;59(3):206-2011. Disponible en: https://www.medigraphic.com/pdfs/revinvcli/ nn-2007/nn073f.pdf

[12] Elmajdoub A, Garbaj A, Abolghait S, ElMahmoudy A. Evaluation of boldenone as a growth promoter in broilers: Safety and meat quality aspects. J Food Drug Anal [Internet]. 2016;24(2):284-92. Disponible en: http://dx.doi. org/10.1016/j.jfda.2015.12.001

[13] Mohammed HH, Badawi ME, El-Tarabany MS, Rania M. Effects of boldenone undecylenate on growth performance, maintenance behaviour, reproductive hormones and carcass traits of growing rabbits. Pol J Vet Sci [Internet]. 1 de junio de 2016;19(2):245-51. Disponible en: http:// journals.pan.pl/dlibra/publication/120972/ edition/105373/content

[14] Tousson E, Mohamed S, El-Gerbed A, Shaleby S. Effects of maturity on histopathological alteration after a growth promoter boldenone injection in rabbits. J Am Sci [Internet]. 2017;7(12):1074-80. Disponible en: http:// www.jofamericanscience.org/journals/am-sci/ am0712/136_7747am0712_1074_1080.pdf

[15] Thabet N, Ezzat A, Haridy M, Kassab $A$, Hamdon H. Safety range of Boldenone Undecylenate injection in beef bulls. Biosci Res [Internet]. 2019;16(2):1556-64. Disponible en: https://www.isisn.org/BR16(2)2019/1556-156416(2)2019BR19-148.pdf

[16] Viljanto MJ, Kicman AT, Walker CJ, Parkin MC, Wolff K, Pearce CM, et al. Elucidation of the biosynthetic pathways of boldenone in the equine testis. Steroids [Internet]. junio de 2019;146:7991. Disponible en: https://linkinghub.elsevier. com/retrieve/pii/S0039128X19300625

[17] Gray B, Tuckley L, Cutler C, Biddle S, Hudson $S$, Gower $S$, et al. Investigations into the analysis of intact drug conjugates in animal sport doping control - Development and assessment of a rapid and economical approach for screening greyhound urine. Drug Test Anal [Internet]. 21 de febrero de 2020;Epub ahead:Epub ahead of print. Disponible en: https://onlinelibrary.wiley.com/ doi/abs/10.1002/dta.2779

[18] Destrez B, Bichon E, Rambaud L, Courant F, Monteau F, Pinel G, et al. Criteria to distinguish between natural situations and illegal use of boldenone, boldenone esters and boldione in cattle. Steroids [Internet]. octubre de 2009;74(1011):803-8. Disponible en: https://linkinghub. elsevier.com/retrieve/pii/S0039128X09001044

[19] Chiesa L, Pasquale E, Panseri S, Cannizzo FT, Biolatti B, Pavlovic R, et al. Pseudoendogenous presence of $\beta$-boldenone sulphate and glucuronide in untreated young bulls from the food chain. Food Addit Contam Part A [Internet]. 3 de junio de 2015;32(6):825-32. Disponible en: http://www. tandfonline.com/doi/full/10.1080/19440049.201 5.1027965

[20] Scarth J, Akre C, van Ginkel L, le Bizec B, de Brabander H, Korth W, et al. Presence and metabolism of endogenous androgenic-anabolic steroid hormones in meat-producing animals: A review. Food Addit Contam - Part A Chem Anal Control Expo Risk Assess. 2009;26(5):640-71.

[21] Jiang J, Zhang H, Li G, Yang X, Li R, Wang $\mathrm{Z}$, et al. Establishment and Optimization of Monoclonal Antibody-based Heterologous dcELISA for 19-Nortestosterone Residue in Bovine Edible Tissue. J Food Sci. 2012;77(4).

[22] Matraszek-Zuchowska I, Woźniak B, Kłopot A, Witek S, Sielska K, Posyniak A. Control of anabolic hormone residues in tissues of slaughter animals in Poland during the period of 2011-2015. J Vet Res. 2017;61(1):69-79.

[23] Celis De La Rosa A, Labrada V. Bioestadística [Internet]. Tercera Ed. Editorial El Manual Moderno SA de CV, editor. México DF; 2014. 6370 p. Disponible en: https://www.academia. edu/21856685/Celis_De_La_Rosa_Alfredo_Y_ Labrada_Vanessa_-_Bioestadistica_3ed_

[24] Luengo J, Aros C, Gómez L. Determinación de la edad del bovino según las características morfológicas de los dientes incisivos. Contribución a la aplicación de la norma chilena 1423 Of. 84. Terminología y clasificación. Av en Ciencias Vet [Internet]. 1990;5(1). Disponible en: https:// 
avancesveterinaria.uchile.cl/index.php/ACV/ article/view/10398

[25] Braña D, Ramirez E, Rubio M, Sánchez A, Torrescano G, Arenas M, et al. Manual de análisis de calidad en muestras de Carne [Internet]. Primera Ed. Querétaro-México: Centro Nacional de Investigación Disciplinaria en Fisiología y Mejoramiento Animal; 2011. Disponible en: https://www.academia.edu/22857285/._Manual_ de_Análisis_de_Calidad_en_Muestras_de_Carne [26] Biooscientific. MaxSignal® Boldenone ELISA Test Kit [Internet]. Austin-USA: PerkinElmer, Inc; 2019. Disponible en: http://www.medibena. at/media/bioo/product-sheets/1085-01+MaxSig nal+Boldenone+ELISA+Test+Kit+Manual.pdf

[27] Reig M, Toldrá F. Veterinary drug residues in meat: Concerns and rapid methods for detection. Meat Sci [Internet]. enero de 2008;78(1-2):60-7. Disponible en: https://linkinghub.elsevier.com/ retrieve/pii/S0309174007002719

[28] Sumano H, Ocampo L. Farmacología Veterinaria [Internet]. Tercera Ed. México DF: McGraw Hill; 2006. 361-406 p. Disponible en: https://es.scribd.com/doc/55938774/ Farmacologia-Veterinaria-Tercera-EdicionSumano-Ocampo

[29] Bolaños T, Inga R. Evaluación de la ganancia de peso en bovinos Charolais mediante la aplicación de dos anabólicos (Revalor G y Boldenona) frente a animales castrados en la provincia de Morona Santiago [Internet]. Universidad Politécnica Salesiana-Sede Cuenca; 2010. Disponible en: https://dspace.ups.edu.ec/ bitstream/123456789/1089/14/UPS-CT001999. pdf

[30] Talero V, Medina Ó, Rozo-Núñez W. Técnicas analíticas contemporáneas para la identificación de residuos de sulfonamidas, quinolonas y cloranfenicol. Univ Sci [Internet]. 2014;19(1):1128. Disponible en: https://revistas.javeriana.edu. co/index.php/scientarium/article/view/6618

[31] Vargas MB. Evaluación de la presencia de los esteroides boldenona y trembolona en músculo bovino de la Provincia de Manabí [Internet]. Universidad Tecnológica Equinoccial; 2018. Disponible en: http://repositorio.ute.edu.ec/ bitstream/123456789/16742/1/70144_1.pdf

[32] ICA, INVIMA. Informe de resultados del Plan Nacional Subsectorial de Vigilancia y Control de Residuos de Medicamentos Veterinarios y Contaminantes Químicos en Carne Bovina 2015 - 2016 [Internet]. Bogotá-Colombia; 2016. Disponible en: https://www.invima.gov. co / d ocuments / 20143 / 430365 / I N FO R MEBOVINOS-2015-2016.pdf

[33] Casati S, Ottria R, Ciuffreda P. 17 $\alpha$ - and
17 $\beta$-boldenone 17-glucuronides: Synthesis and complete characterization by $1 \mathrm{H}$ and $13 \mathrm{C}$ NMR. Steroids. 2009;74(2):250-5.

[34] Granja RHMM, Salerno AG, De Lima AC, Montalvo C, Reche KVG, Giannotti FM, et al. Liquid chromatography/tandem mass spectrometry method to determine boldenone in bovine liver tissues. J AOAC Int. 2014;97(5):1476-80.

[35] Leporati M, Bergoglio M, Capra P, Bozzetta E, Abete MC, Vincenti M. Development, validation and application to real samples of a multiresidue LC-MS/MS method for determination of $\beta 2$ agonists and anabolic steroids in bovine hair. J Mass Spectrom. 2014;49(9):936-46.

[36] Zhang J-N, Yang L, Zhang M, Liu Y-S, Zhao J-L, He L-Y, et al. Persistence of androgens, progestogens, and glucocorticoids during commercial animal manure composting process. Sci Total Environ [Internet]. mayo de 2019;665:919. Disponible en: https://linkinghub.elsevier.com/ retrieve/pii/S0048969719306023 